\title{
Laser-like Instabilities in Quantum Nano-electromechanical Systems
}

\author{
S. D. Bennett and A. A. Clerk \\ Department of Physics, McGill University, Montréal, Québec, Canada, H3A 2T8
}

(Dated: Sept. 18, 2006)

\begin{abstract}
We discuss negative damping regimes in quantum nano-electromechanical systems formed by coupling a mechanical oscillator to a single-electron transistor (normal or superconducting). Using an analogy to a laser with a tunable atom-field coupling, we demonstrate how these effects scale with system parameters. We also discuss the fluctuation physics of both the oscillator and the single-electron transistor in this regime, and the degree to which the oscillator's motion is coherent.
\end{abstract}

PACS numbers:

At their heart, quantum nano-electromechanical systems (NEMS) consist of nothing more than a mechanical oscillator coupled to a mesoscopic conductor. Despite their apparent simplicity, they have been the focus of considerable recent interest. It has been predicted and shown experimentally that a single-electron transistor (SET) coupled to a mechanical oscillator may be used for near quantum-limited position detection ${ }^{1.2 .3 .4 .5}$. An equally fascinating aspect of these systems is their underlying quantum dissipative physics: from the point of view of the oscillator, the mesoscopic conductor acts as a non-equilibrium bath which both heats and damps the oscillator ${ }^{6.7 .8}$. For a sufficiently weak oscillatorconductor coupling, and a sufficiently high-Q oscillator, one can map the conductor onto an effective equilibrium bath. Even in this regime, novel effects may take place. Recent work shows that in systems with either normal-metal or superconducting SET's, one can operate in regimes where the SET generates negative damping of the oscillator ${ }^{9,10,11}$. The resulting instability is ultimately cut off by a nonlinearity in the dynamics, and is characterized by an effective strong coupling between the mechanical and electronic dynamics.

In this paper, we investigate the properties of both normal-state and superconducting SET NEMS systems in the negative damping regime. Using the analogy between these systems and a laser with a tunable atom-field coupling, we derive simple scaling relations for the stationary state and fluctuations in the negative damping regime. We also discuss how noise measurements could be used to sensitively probe the physics in this regime. In particular, the critical slowing down associated with the transition to the "lasing" state can be seen clearly in the low-frequency current noise of the transistor. Note that the analogy between laser physics and a superconducting SET NEMS was also studied recently in Ref. 12 using a numerical approach. An alternate proposed mechanical analogue of a laser was discussed in Ref. 13 .

Model. We consider a standard SET-based NEMS where the oscillator acts as a voltage gate with an $x$ dependent coupling capacitance to the SET island; details may be found in Ref. 5. In the absence of any intrinsic damping of the oscillator, the total Hamiltonian is given by $H=H_{o s c}+H_{0}+H_{C}$. Here, $H_{o s c}$ describes an oscillator with angular frequency $\Omega$ and mass $m$. $H_{0}$ de- scribes the non-interacting part of the SET Hamiltonian, and includes the kinetic energies of electrons in the leads and island, as well as tunnelling terms taking electrons to and from the leads; in the case of a superconducting SET (SSET), the island and leads are described by BCS Hamiltonians. Finally, $H_{C}$ describes the Coulomb charging energy of the island in the presence of the oscillator:

$$
H_{C}=E_{C}\left[\hat{n}^{2}-2 \hat{n}\left(\mathcal{N}_{0}+\frac{A}{2 E_{C}} \hat{x}\right)\right],
$$

where $E_{C}$ is the charging energy of the SET island, $\hat{n}$ is the island charge, and $\mathcal{N}_{0}$ is the dimensionless gate voltage associated with a fixed control gate. The coupling strength is given by $A / 2 E_{C}=\left(V_{o s c} / e\right) \cdot d C_{o s c} / d x$, where $V_{o s c}$ is the voltage bias on the oscillator and $C_{o s c}$ is the capacitance between the oscillator and the SET island 11 . In what follows, the oscillator is always in the regime where $C_{o s c}$ depends linearly on $x$. We see that $\hat{n}$ acts as a back-action force on the oscillator, while the SET experiences an effective $x$-dependent gate voltage $\mathcal{N}[x]=\mathcal{N}_{0}+\left(A / 2 E_{C}\right) \cdot x$. In the usual case where $\Omega$ is much smaller than the typical tunnelling rates in the SET, and the coupling strength $A$ is sufficiently weak, one can combine linear-response theory with a BornOppenheimer approximation to rigorously derive a classical Langevin equation describing the oscillator ${ }^{11}$ :

$$
m \ddot{x}=-(k+\Delta k[x]) x-m\left(\gamma_{0}+\gamma[x]\right) \dot{x}+\delta f_{0}+\delta f[x] .
$$

The back-action force leads to a damping term $\gamma[x]$, spring-constant renormalization $\Delta k[x]$, and a stochastic force $\delta f(t)$ with a white, $x$-dependent spectral density $S_{\delta f}[x]$. The damping and noise are determined by the quantum noise spectrum $S_{n}(\omega, \mathcal{N}) \equiv$ $\int d t e^{i \omega t}\langle\hat{n}(t) \hat{n}(0)\rangle_{\mathcal{N}}$ of the SET island charge fluctuations, evaluated at zero coupling to the oscillator and for a fixed gate voltage $\mathcal{N} ; \Delta k$ is determined by the corresponding charge susceptibility:

$$
\begin{aligned}
m \gamma[x] & =\left.\frac{A^{2}}{\hbar} \frac{d S_{n}(\omega, \mathcal{N})}{d \omega}\right|_{\omega=0, \mathcal{N}=\mathcal{N}[x]}, \\
S_{\delta f}[x] & =A^{2} S_{n}(\omega=0, \mathcal{N}[x]), \\
\Delta k[x] & =\left.\frac{A^{2}}{2 E_{C}} \frac{d\langle n\rangle}{d \mathcal{N}_{0}}\right|_{\mathcal{N}=\mathcal{N}[x]} .
\end{aligned}
$$


The spectrum $S_{n}(\omega, \mathcal{N})$ can been calculated for various SET processes using standard techniques 11 . Physically, Eqs. (3) -57 are based on the approximation that at each instant in time, the oscillator sees the SET as an effective bath whose properties are determined by the instantaneous gate voltage $\mathcal{N}[x]$. The separation of timescales required for this description to be valid is well satisfied in current experiments ${ }^{2.3 .4}$. Eq. (2) also includes damping and noise terms due to coupling to an equilibrium bath: in the absence of the SET, the intrinsic quality factor of the oscillator is $Q_{0}=\Omega / \gamma_{0}$ and its temperature $T_{0}$ is determined by the strength of the force $\delta f_{0}(t)$.

Negative Damping. As the SET is voltage biased and hence out of equilibrium, it is possible for the back-action damping $\gamma$ to become negative and even overwhelm the positive bath damping $\gamma_{0}$. For a SSET, negative damping can arise near operating points corresponding to incoherent Cooper-pair tunneling in the single/double Josephson quasiparticle processes (JQP/DJQP) $\stackrel{10,11}{ }$. If SET voltages are chosen so that tunneling Cooper-pairs can move closer to resonance by emitting energy to the oscillator, one generically gets negative damping. Negative damping can also arise in a normal metal SET if one has strongly energy-dependent tunnel matrix elements and a resulting non-monotonic $\langle n\rangle$ versus $\mathcal{N}$ curve $e^{9.11}$.

For either of the above two cases, negative damping means that the stationary state of the oscillator will be determined by the nonlinearity of the SET-induced damping (i.e. the fact that $\gamma$ is a function of $x$ ). Crudely speaking, the stationary state corresponds to an oscillator amplitude large enough that the oscillating gate voltage $\mathcal{N}[x]$ smears out the negative damping from the SET, making the total damping zero. A convenient way to describe this physics is to make use of the high quality factors of typical NEMS. One can then derive a Kramerslike equation for the energy distribution function $w(E, t)$ of the oscillator ${ }^{9.11}$ :

$$
\frac{d}{d t} w=\frac{\partial}{\partial E} E\left[\gamma_{0}+\gamma(E)+\frac{D_{0}+D(E)}{m} \frac{\partial}{\partial E}\right] w,
$$

where the energy-dependent damping and diffusion are given by

$$
\begin{aligned}
\gamma(E) & =2 \int_{0}^{2 \pi} \frac{d \theta}{2 \pi} \gamma[x] \cos ^{2} \theta, \\
D(E) & =\int_{0}^{2 \pi} \frac{d \theta}{2 \pi} S_{\delta f}[x] \cos ^{2} \theta,
\end{aligned}
$$

with $x=\sqrt{2 E / k} \sin \theta$. The stationary solution of Eq. (66) is given by a generalized Boltzmann distribution. Defining $\widetilde{T}_{o s c}(E) \equiv\left[D(E)+D_{0}\right] /\left[\gamma(E)+\gamma_{0}\right]$, we have

$$
w(E) \propto \exp \left[-\int_{0}^{E} \frac{d E^{\prime}}{k_{B} \widetilde{T}_{o s c}\left(E^{\prime}\right)}\right] .
$$

In the negative damping regime of interest, $\gamma(E)+\gamma_{0}$ is negative at $E=0$, and becomes positive at large enough
$E$. In the simplest case, $\gamma(E)$ is simply a monotonic increasing function of $\mathrm{E}$; this arises in a SSET if one detunes the Cooper-pair resonances to achieve a maximum negative damping ${ }^{11}$. One then finds that $w(E)$ has a maximum at $E=E_{0}$, where $E_{0}$ is defined by $\gamma\left(E_{0}\right)+\gamma_{0}=0$. Moreover, if $E_{0}$ is sufficiently large, the distribution has a Gaussian form, with a width given by

$$
\sigma^{2}=\frac{D_{0}+D\left(E_{0}\right)}{m}\left[\left.\frac{d \gamma}{d E}\right|_{E=E_{0}}\right]^{-1} .
$$

The negative damping instability discussed here in the context of a quantum NEMS is reminiscent of the physics of a laser. In the typical case of a single mode cavity laser with fast atomic relaxation, the effects of the populationinverted atoms on the relevant cavity mode can be described in terms of amplitude-dependent damping that is negative for small mode occupancies 14 . In our case, it is instead tunnelling electrons or Cooper pairs in the SET which provide the negative damping. Note also that our system is also very similar to a non-resonantly driven oscillator coupled non-linearly to an oscillator bath; the negative damping regime here was studied extensively (both classically and quantum mechanically) by Dykman and Krivoglaz ${ }^{15}$ In what follows, we explore in more detail the extent to which our system is analogous to a laser.

Scaling. Two basic features of a laser are both a large average number of quanta in the lasing mode, and relatively small number fluctuations described by Poisson statistics. We would thus like to know how these quantities scale with system parameters in the SET-based NEMS considered here. We first assume that the SET gate voltage $\mathcal{N}_{0}$ has been set to maximize the negative damping. We then introduce the characteristic scale for the gate voltage, $\mathcal{N}^{*}$, which is defined as the change of the gate voltage required to significantly reduce the magnitude of the SET damping 19 . For example, in the case of incoherent Cooper-pair tunneling ${ }^{4}, \mathcal{N}^{*}$ is naturally defined as the change of $\mathcal{N}$ needed take the SET off resonance. This yields $2 E_{C} \mathcal{N}^{*} \sim \hbar \Gamma$, where $1 / \Gamma$ is the lifetime of the Cooper-pair resonance.

The gate voltage scale $\mathcal{N}^{*}$ can be translated into an energy scale $E^{*}$ via

$$
E^{*}=2 k\left(\frac{E_{C} \mathcal{N}^{*}}{A}\right)^{2} .
$$

This is the approximate energy needed by the oscillator to completely smear out the negative damping contribution of the SET. As we will see, $E^{*}$ is not the same as the energy $E_{0}$ discussed above.

Next, we write the SET damping as $\gamma(E)=$ $-\gamma_{\max } g\left(E / E^{*}\right)$, where $g(0)=1$, and $\gamma_{\max }=-\gamma(0)$ is the maximum of the negative SET damping. For simplicity, we assume $\mathrm{g}$ is monotonic decreasing, as is the case for an optimally tuned SSET. The most probable oscillator energy, i.e. the energy at which a maximum 
occurs in $w(E)$, is then given by

$$
\begin{aligned}
E_{0} & =E^{*} \cdot g^{-1}\left(\frac{\gamma_{0}}{\gamma_{\max }}\right) \\
& =K \cdot Q_{0} \hbar \Omega \cdot\left[\frac{\gamma_{0}}{\gamma_{\max }}\right] g^{-1}\left(\frac{\gamma_{0}}{\gamma_{\max }}\right) .
\end{aligned}
$$

where $Q_{0}=\Omega / \gamma_{0}$ is the intrinsic oscillator quality factor, and the dimensionless constant $K$ is independent of the oscillator-SET coupling strength:

$$
K=\left.\frac{2\left(E_{C} \mathcal{N}^{*}\right)^{2}}{\hbar^{2}} \frac{d S_{n}}{d \omega}\right|_{\omega=0, \mathcal{N}=\mathcal{N}_{0}} .
$$

One can easily estimate the parameter $K$ for a given SET operating point. For example, we have already shown that $2 E_{C} \mathcal{N}^{*} \simeq \hbar \Gamma$ for the DJQP process in a SSET. Further, a simple estimate (which is born out by the full calculation in Ref. 11) gives $d S_{n} / d \omega \simeq\left(\hbar / E_{J}\right)^{2}$ where $E_{J}$ is the Josephson energy for the SET (both junctions are assumed equal). We thus have $K \simeq\left(\hbar \Gamma / E_{J}\right)^{2}$. For the experiments of Ref. 4, this yields $K \simeq 6$.

Using Eq. (13), we find that we can maximize the average oscillator energy $E_{0}$ with respect to the coupling strength ${ }^{20}$. In that equation, we see that the coupling strength only enters $E_{0}$ through the ratio $\gamma_{0} / \gamma_{\max }$. Moreover, as $g$ is a monotonic decreasing function, the same is true of $g^{-1}$. It thus follows that $E_{0}$ must have a maximum as a function of the coupling. Physically, this is easy to understand. For too weak a coupling, the intrinsic damping $\gamma_{0}$ dominates the effects of the oscillator, and there is no instability. For too strong a coupling, the oscillator does not need much energy to smear out the effects of the SET-induced damping.

Finally, we define $b$ to be the maximum of the function $y g^{-1}(y)$, and define $y_{0}$ to be the value of $y$ at which this function is maximized. With our choice of $E^{*}$, both $b$ and $y_{0}$ are of order unity. We thus have ${ }^{21}$

$$
\begin{aligned}
{\left[E_{0}\right]_{\text {max }} } & =b \cdot K Q_{0} \hbar \Omega, \\
A_{\text {opt }} & =\sqrt{\frac{1}{y_{0} \cdot K Q_{0}}} \frac{E_{C} \mathcal{N}^{*}}{\Delta x},
\end{aligned}
$$

where $\Delta x=\sqrt{\hbar /(2 m \Omega)}$ is the zero-point uncertainty in the oscillator position.

We thus see that for an optimal coupling strength, the average oscillator energy scales as the intrinsic oscillator quality factor. For sufficiently large $Q_{0}$, one can indeed have a high mode occupancy in the resonator. Also note that the optimal coupling strength scales as $1 / \sqrt{Q_{0}}$. One can show along the same lines that in general, $E_{0} / Q_{0}$ only depends on the coupling and the intrinsic quality factor through the combination $A \sqrt{Q_{0}}$. The validity of these scaling relations have been tested against numerical solution of Eq. (6) for both JQP and DJQP processes ${ }^{16}$.

To further the analogy to a laser, we must also characterize energy fluctuations in the stationary state. At the optimal coupling strength, Eq. (13) yields the exact result $\left.\frac{d \gamma}{d E}\right|_{E_{0}}=\gamma_{0} / E_{0}$; using this and assuming that the bath temperature is low enough that the SET dominates the oscillator diffusion, from Eq. (10) we find

$$
\left\langle\left\langle E^{2}\right\rangle\right\rangle=\frac{\left(D\left(E_{0}\right)\right)}{m \gamma_{0}} \times E_{0}=k_{B} T^{*} \cdot E_{0} .
$$

As in a laser, the variance of $E$ scales as $\sqrt{\langle E\rangle}$; using Eq. (15), we see that the relative energy fluctuations become small as $1 / \sqrt{Q_{0}}$. The degree to which the energy fluctuations are super-Poissonian is determined by the effective temperature $T^{*}$ defined above. In practice, $T^{*}$ will be on the order of the SET effective temperature at $E=0$, defined by $k_{B} T_{\text {eff }}=D(0) / m|\gamma(0)|$. Using the definition of $\gamma$ and $D$ in terms of $S_{n}(\omega)$, one can show that in the regime we consider (where the SET is much faster than the oscillator), $T_{\text {eff }}$ and $T^{*}$ must both be much larger than $\hbar \Omega$. Our system is thus more like a maser than a laser: the number fluctuations are far greater than the bound set by purely quantum noise.

Phase fluctuations and linewidth. Another hallmark of a laser is its narrow linewidth, which is limited by relatively weak phase fluctuations in the stationary state ${ }^{17}$. It is thus interesting to ask about phase fluctuations in our system. A convenient approach is to work directly with Eq. (2), and make a rotating-wave approximation, where one keeps track of the oscillator amplitude and the slowly varying part of the oscillator phase. Focusing on fluctuations about the stationary, large-amplitude lasing state, we start by writing

$$
\dot{x}(t)-i \tilde{\Omega} x(t)=\left[\rho_{0}+\delta \rho(t)\right] e^{-i \tilde{\Omega} t} e^{i \phi(t)},
$$

where $\rho_{0}=\sqrt{2 E_{0} / m}$ is the stationary amplitude of the oscillator, and $\tilde{\Omega}=\sqrt{\left(k+\Delta k\left(E_{0}\right) / m\right.}$ is the renormalized frequency of the oscillator, with

$$
\Delta k(E)=2 \int_{0}^{2 \pi} \frac{d \theta}{2 \pi} \Delta k[x] \sin ^{2} \theta .
$$

The linewidth of the oscillator will be determined by the fluctuations in the oscillator phase, $\phi(t)$. Linearizing Eq. (2) in $\delta \rho$ and $d \phi / d t$, and coarse graining over a timescale long compared to $1 / \Omega$, we find that the spectral density of this fluctuating phase is

$$
S_{\phi}(\omega)=\frac{2 D_{\phi}}{\omega^{2}}\left[1+\frac{\alpha^{2}}{1+\left(\omega / \gamma_{0}\right)^{2}}\right],
$$

where the intrinsic phase diffusion constant is given by

$$
D_{\phi}=\frac{1}{4 m E_{0}} \int_{0}^{2 \pi} \frac{d \theta}{2 \pi} S_{\delta f}[x] \sin ^{2} \theta .
$$

Here, $\alpha$ characterizes the relative importance of the coupling between amplitude and phase fluctuations:

$$
\alpha=\frac{d \Omega / d E}{d \gamma / d E} \sqrt{\frac{D\left(E_{0}\right) / m}{E_{0} D_{\phi}}} \sim \frac{1}{m \Omega} \frac{d(\Delta k) / d E}{d \gamma / d E},
$$


where the derivatives are evaluated at $E_{0}$, and we have used the fact that up to factors of order unity, $D_{\phi} \sim$ $D\left(E_{0}\right) /\left(m E_{0}\right) . \alpha$ is analogous to Henry's $\alpha$ parameter in standard laser theory ${ }^{18}$; on a physical level, energy fluctuations lead to additional frequency fluctuations through the SET spring constant modulation $\Delta k[x]$.

In a semiclassical picture, the SET-induced damping is due to the small but finite response time $\tau$ of the SET to the oscillator's motion ${ }^{11}$; one can write $m \gamma \sim(\Delta k) \tau$. From Eq. (22), one then has $\alpha \sim 1 /(\Omega \tau) \gg 1$, as we are explicitly considering a SET which is much faster than the oscillator. This means that unlike a typical laser, the linewidth of the oscillator in the stationary state of our "lasing" NEMS is set by the coupling between amplitude and phase fluctuations. The crucial difference from a laser is that in the NEMS case, the transfer of energy between the oscillator and the SET is not resonant, whereas in a laser the coupling between the mode and the atoms is resonant. Thus, in a laser, one obtains a damping force from the atoms even if they are assumed to respond instantaneously to the mode; in contrast, there would be no back-action damping in our NEMS in this limit.

Finally, for sufficiently large $E_{0}$ we find that the oscillator position noise spectrum exhibits a Lorentzian form centered on $\omega=\tilde{\Omega}$ with a width $\gamma_{e f f}$ determined by the zero-frequency limit of $S_{\phi}$. Up to factors of order unity, we find that this width can be much narrower than that set by the intrinsic damping of the oscillator:

$$
\frac{\gamma_{e f f}}{\gamma_{0}} \sim \frac{k_{B} T^{*}}{4 E_{0}} \alpha^{2} \propto \frac{1}{K Q_{0}} .
$$

Critical slowing down and shot noise. As was discussed in Ref. 9, the instability physics discussed here will manifest itself directly in the shot noise of the SET. In the stationary state, the oscillator's motion is large enough that the resulting oscillations in the SET gate voltage $\mathcal{N}$ strongly modulate the current. One consequence is that the slow energy dynamics of the SET will lead to long-time correlations of tunnel events in the SET, and thus a large enhancement of the low-frequency current noise. The magnitude of this enhancement is expected to be inversely proportional to the relaxation rate of energy fluctuations in the oscillator, $\lambda_{E}$ : this represents the time over which tunnel events are correlated. At the optimal coupling, a linearized treatment of fluctuations (as above) shows that $\lambda_{E}=\gamma_{0}$. Moreover, as a function of coupling strength, $\lambda_{E}$ exhibits critical slowing down: it reaches a minimum as the coupling strength is tuned through the bifurcation between small-amplitude and large-amplitude states of the oscillator. Without noise, this occurs at the coupling voltage $V_{\text {crit }}$ for which $\gamma(E=0)+\gamma_{0}=0$; fluctuations push the transition to a slightly higher coupling voltage ${ }^{17}$. We thus expect a maximum shot noise enhancement near $V_{\text {osc }}=V_{\text {crit }}$.

We have numerically calculated the expected shot noise enhancement for a NEMS system operated near the DJQP resonance, using parameters from Ref. 4. This can be calculated in a straightforward manner from the

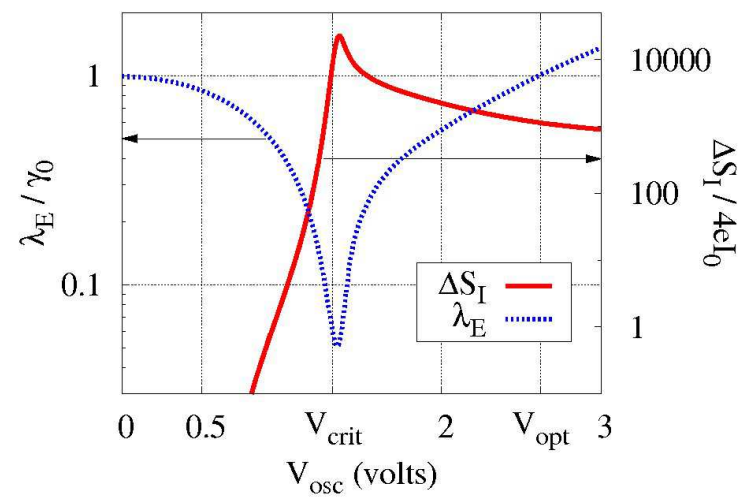

FIG. 1: Signatures of critical slowing down for the DJQP process in a SSET. Dotted line (left vertical axis): energy relaxation rate $\lambda_{E}$ versus coupling voltage $V_{o s c}$. The minimum near $V_{\text {crit }}$ corresponds to critical slowing down, and one finds $\lambda_{E}=\gamma_{0}$ at the optimal coupling voltage, $V_{o p t}$. Solid line (right vertical axis): zero-frequency current noise enhancement $\Delta S_{I}$ versus $V_{o s c}$, scaled by the current noise of the uncoupled SSET. We have used device parameters from Ref. 4; for the SSET, these are $E_{C}=175 \mu \mathrm{eV}, \Delta=192 \mu \mathrm{eV}, g=0.5$, and $E_{J}=15 \mu \mathrm{eV}$. For the oscillator: $\Omega=22 \mathrm{MHz}, k=10$ $\mathrm{N} / \mathrm{m}$ and $Q_{0}=120000$. We have also used $T_{0}=20 \mathrm{mK}$ and $A=1.05 \times 10^{-13} \mathrm{~N}$ when $V_{\text {osc }}=1$ volt.

energy diffusion equation Eq. (6) ${ }^{9}$. Fig. 11 shows a clear maximum in the shot noise as a function of the coupling voltage, which corresponds to the critical slowing down. We stress that the maximum does not occur at the optimal coupling voltage $V_{\text {opt }}$, but rather near the voltage $V_{\text {crit }}$ corresponding to the bifurcation.

In conclusion, we have shown how negative damping instabilities in SET NEMS scale with system parameters, and have investigated the amplitude and phase fluctuations in the stationary state. This work was supported by NSERC and FQRNT.
1 M. P. Blencowe and M. N. Wybourne, Appl. Phys. Lett. 77, 3845 (2000).

2 R. G. Knobel and A. N. Cleland, Nature (London) 424, 291 (2003).

3 M. D. LaHaye, O. Buu, B. Camarota, and K. C. Schwab, Science 304, 74 (2004).
4 A. Naik, O. Buu, M. D. LaHaye, A. D. Armour, A. A. Clerk, M. P. Blencowe, and K. C. Schwab, Nature (London) 443, 193 (2006).

5 M. P. Blencowe, Phys. Rep. 395, 159 (2004).

6 D. Mozyrsky and I. Martin, Phys. Rev. Lett. 89, 018301 (2002). 
7 A. D. Armour, M. P. Blencowe, and Y. Zhang, Phys. Rev. B 69, 125313 (2004).

8 A. A. Clerk, Phys. Rev. B 70, 245306 (2004).

9 Ya. M. Blanter, O. Usmani, and Yu. V. Nazarov, Phys. Rev. Lett. 93, 136802 (2004); ibid., Phys. Rev. Lett. (erratum) 94, 049904 (2005); O. Usmani, Ya. M. Blanter, and Yu. V. Nazarov, cond-mat/060317

10 M. P. Blencowe, J. Imbers, and A. D. Armour, New J. Phys. 7, 236 (2005).

11 A. A. Clerk and S. Bennett, New J. Phys. 7, 238 (2005).

12 D. A. Rodrigues and A. D. Armour, cond-mat/0608166 (2006).

13 I. Bargatin and M. L. Roukes, Phys. Rev. Lett. 91, 138302 (2003).

14 H. Haken, Rev. Mod. Phys. 47, 67 (1975).

15 M. I. Dykman and M. V. Krivoglaz, in Soviet Physics Reviews, edited by I. M. Khalatnikov (Harwood Academic,
1984), vol. 5, pp. 265-441.

16 S. Bennett, Master's thesis, McGill University (2006).

17 M. Lax, in Brandeis Summer Institute of Theoretical Physics Lectures (Gordon and Breach, 1966).

18 C. H. Henry, IEEE J. Quantum Electron. 18, 259 (1982).

19 The precise definition chosen for the scale $\mathcal{N}^{*}$ is not important. Changing $\mathcal{N}^{*}$ by a factor of order unity only changes $b, y_{0}$ and $K$ by a similar factor; the scaling relations of Eqs. (15) and (16) are otherwise unchanged.

20 The average energy $\langle E\rangle$ is slightly larger than $E_{0}$ due to fluctuations; this small difference plays no role here.

${ }^{21}$ In the limit $Q_{0} \rightarrow \infty$, we find $\left[E_{0}\right]_{\max } \rightarrow \infty$ and $A_{\text {opt }} \rightarrow 0$. This poses no contradiction with the work of Blanter et $a l^{\frac{9}{2}}$, who considered $Q_{0} \rightarrow \infty$ for finite, fixed $A$, which leads to finite, but not optimal, $E_{0}$. 